\title{
Robust design of a TMD for the vibration serviceability of a footbridge
}

\author{
Klaus Lievens ${ }^{\mathrm{a}, \mathrm{b}, *}$, Geert Lombaert ${ }^{\mathrm{b}}$, Guido De Roeck ${ }^{\mathrm{b}}$, Peter Van den Broeck ${ }^{\mathrm{a}, \mathrm{b}}$ \\ ${ }^{a}$ KU Leuven, Department of Civil Engineering, Technology Campus Ghent, Gebroeders De Smetstraat 1, B-9000 \\ Ghent, Belgium \\ ${ }^{b}$ KU Leuven, Department of Civil Engineering, Kasteelpark Arenberg 40, B-3001 Leuven, Belgium
}

\begin{abstract}
Footbridges are often designed as slender structures, sensitive to human-induced excitation. In the case where the prevailing vibration serviceability requirements are not met, vibration reduction measures such as tuned mass dampers (TMDs) are needed. Both the prediction of the structural response and the design of the TMD rely on the modal parameters of the footbridge. Measurements after construction of footbridges have shown that it is difficult to accurately predict these modal parameters even with detailed finite element models based on structural drawings. Moreover, these parameters evolve in time due to changing environmental conditions and degradation. It is important to take into account these uncertainties in the vibration serviceability assessment as well as in the design of vibration reduction measures.

The present paper proposes a robust optimisation approach for the design of a TMD which accounts for uncertainties in the modal parameters. The aim is to minimise the mass of the TMD, assumed as a measure for the cost, by tuning the mass, stiffness and damping values, while guaranteeing that vibration serviceability is satisfied for a range of possible values of the natural frequency and modal damping. In order to investigate the trade-off between the mass of the TMD (cost) and the level of uncertainty, a multi-interval approach is adopted. The optimal parameters are found to change significantly with the level of uncertainty. The TMD mass and damping increase for a higher level of uncertainty to satisfy the vibration serviceability requirements in all possible cases.
\end{abstract}

Keywords: vibration serviceability assessment, tuned mass damper, human-induced vibrations, robust design, worst-case, multi-interval analysis

\section{INTRODUCTION}

The coming of advanced design methods and high strength materials enables the design of slender footbridges characterised by low natural frequencies, possibly in the range of loading frequencies induced by human walking. Several vibration serviceability problems were reported [1, 2] demonstrating the necessity of an assessment of vibration serviceability in the design stage.

If the predicted vibration levels exceed the threshold for vibration comfort, a tuned mass damper (TMD) can be included as a passive vibration control device [3]. The TMD serves as an energy absorber and is characterised by its mass, stiffness and damping constant. The TMD parameters are tuned to the modal parameters of the main structure to optimise its performance.

${ }^{*}$ Corresponding author. Tel.: +32 (0)9 2658612.

Email address: klaus.lievens@kuleuven.be (Klaus Lievens) 
The vibration serviceability assessment and the performance of the TMD are highly sensitive to deviations in the natural frequency of the main structure which may lead to an ineffective response reduction. A discrepancy between the values of the modal parameters assumed for the design of the TMD and the actual values adversely affects the performance. This effect of detuning was studied by Rana and Soong [4], Werkle [5], Jensen [6] and Petersen [7] while Rizzi [8] investigated the effect of a mismatch between the assumed and actual modal damping. In a recent study by Van Nimmen et al. [9], it was shown that even with detailed finite element models (FEM) based on structural drawings, errors in natural frequency may go up to $10 \%$.

The conventional approach for the design of a TMD as presented by Den Hartog 10 and Asami [11, 12] does not account for uncertainties in the loading, or in the modal parameters of the main system. Several studies considered the optimisation of the performance of a TMD under uncertain conditions. In [13] a conventional TMD design is compared with single and multi-objective robust design optimisations. The analysis showed that the TMD parameters of the conventional and the robust design differ considerably from each other. Zang et al. 114 propose to determine the TMD mass, stiffness and damping by solving a multi-objective optimisation problem to minimise a weighted sum of the mean value and the standard deviation of the peak response. An alternative interpretation in [15] proposes a fuzzy approach and formulates the objective function based on the credibility theory. In these robust optimisation problems, it is generally assumed that only the loads are the uncertain quantities [16]. However, in many problems of structural dynamics, the uncertainty of the system parameters has a larger influence on the response than the uncertainty of the load [17].

The present paper proposes a robust TMD optimisation approach which accounts for uncertainties on the modal parameters of the main structure to guarantee performance in a range of the natural frequency and modal damping. Furthermore, in the proposed TMD parameter tuning a load model to account for the effect of the human-induced walking load is assumed. A multiinterval approach is proposed to investigate how the uncertainty for the natural frequency and modal damping affects the TMD parameters and performance. At different levels of uncertainties, the TMD mass, which is assumed to be a measure for the TMD cost, is minimised by tuning the TMD mass, stiffness and damping constant. Robustness is obtained by considering the vibration serviceability performance for multiple pedestrian densities and a range of possible values of the modal parameters for different levels of uncertainty. This ensures that the vibration serviceability criterion is always met instead of minimising the sensitivity of the performance to a combination of the expected value and standard deviation of the response as is commonly done. The multi-level robust optimisation approach is illustrated for the Phénix footbridge in Charleroi, Belgium. The TMD parameters are tuned with constraints limiting the vibration level as well as the relative displacement between the TMD and the bridge, to ensure correct operation of the device. By comparing the design parameters for different ranges of uncertainty of the natural frequency and modal damping of the main structure, the trade-off between cost and robustness is investigated.

The structure of the paper is as follows. The Phénix footbridge is presented in section 2. A vibration serviceability assessment is made according to the HiVoSS guideline [18] in section 3. In section 4 , design uncertainties are studied by means of a fuzzy analysis considering a variation of the natural frequency and modal damping within a range reasonably expected in design stage. Subsequently in section 5, the parameters of the TMD are determined according to Asami [11]. The TMD is tuned for both a nominal and a worst-case scenario. Finally, section 6 proposes a multiinterval robust design optimisation of the TMD to determine the TMD parameters for different levels of uncertainty. The optimal TMD parameters are evaluated for different levels of uncertainty to investigate their efficiency. 


\section{THE PHÉNIX FOOTBRIDGE}

The Phénix footbridge is a slender construction with a single span of $38.25 \mathrm{~m}$ and a width of $13.35 \mathrm{~m}$ and is situated near the railway station of Charleroi (Belgium). The cross section of the bridge consists of three parts. The midspan is a main box of $6.5 \mathrm{~m}$ by $1 \mathrm{~m}$ with, on both sides, cantilevered tapering I-profiles that are mounted on fixed distances. Figures 1(a) and 1(b) show a global view of the footbridge and the cross section of the structure, respectively.

The bridge is supported by four neoprene bearings. Vertical translations are fixed at all corners while horizontal translations are fixed at one. An additional support is added in the middle of one side of the bridge to avoid lateral movements. An overview of the support conditions is given schematically in figure $1(\mathrm{c})$.

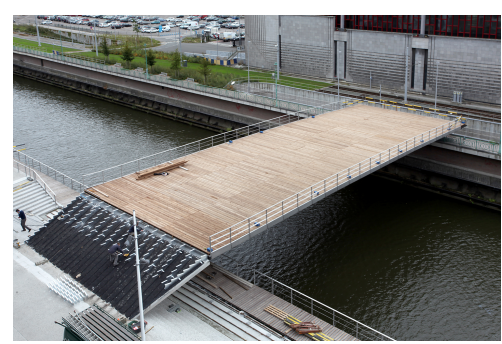

(a)

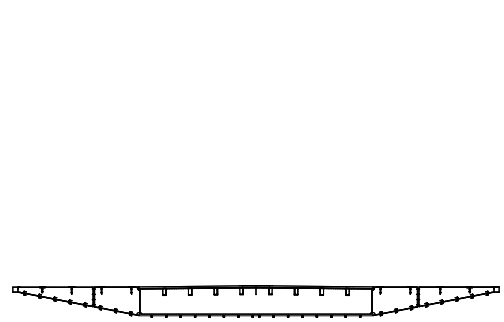

(b)

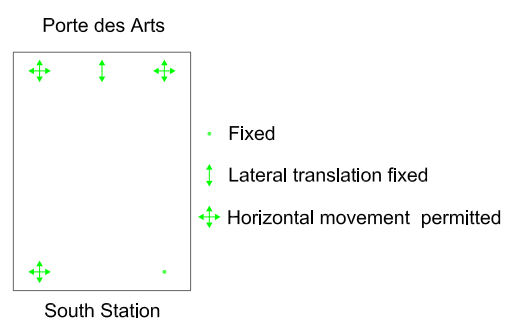

(c)

Figure 1: The Phénix footbridge in Charleroi: (a) Global view, (b) Cross section, (c) Support conditions.

The dynamic behaviour of the construction is defined by its modal parameters. The mode shapes as calculated by a FEM are given in figure 2. The first mode has a natural frequency of 1.65 $\mathrm{Hz}$ which is lower than $5 \mathrm{~Hz}$ and therefore in the range of the loading frequencies of the walking load. The second mode is the second bending mode and the third mode is the first torsional mode.

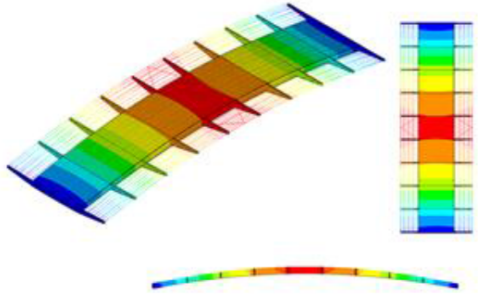

(a)

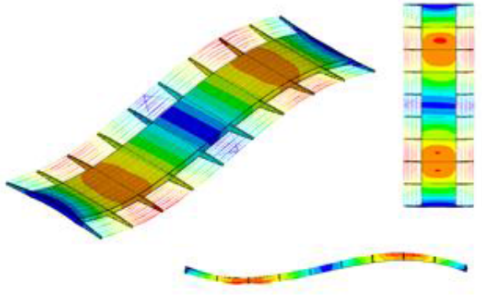

(b)

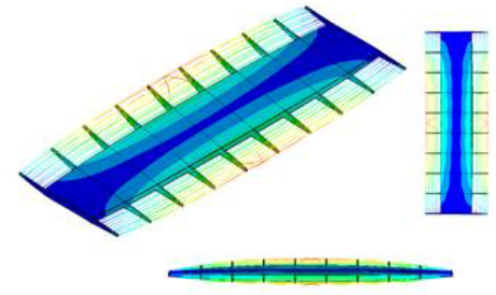

(c)

Figure 2: Modal parameters of the Phénix footbridge based on the FEM: (a) 1st bending mode $\left(f_{1}=1.65 \mathrm{~Hz}\right),(\mathrm{b})$ 2nd bending mode $\left(f_{2}=5.22 \mathrm{~Hz}\right)$ and (c) 1st torsional mode $\left(f_{3}=6.15 \mathrm{~Hz}\right)$

\section{VIBRATION SERVICEABILITY ASSESSMENT}

In the present section, the vibration serviceability assessment is performed according to the HiVoSS design guideline [18]. An essential element in the vibration serviceability assessment of footbridges is the prediction of the human-induced vibrations, requiring a description of the dynamic behaviour of the structure and the crowd-induced loads. The latter can be described by detailed step-by-step simulations whereby a statistical analysis is required to evaluate the induced structural response [19] or, as in the present study, by an equivalent deterministic load derived to represent 
the effect of a crowd. The methodology developed next, however, is general and can be applied using other design guidelines.

\subsection{Response calculation and evaluation}

The considered walking load for a single pedestrian is approximated by a periodic load composed of multiple harmonics of the fundamental step frequency $f_{s}$ and is written as a Fourier series:

$$
F(t)=G+\sum_{h=1}^{n_{h}} G \alpha_{h} \sin \left(2 \pi h f_{s} t-\theta_{h}\right)
$$

with $F(t)$ the walking force in the time domain, $G$ the weight of the pedestrian, $h$ the number of the harmonic load component, $\alpha_{h}$ the dynamic load factor for the $h^{\text {th }}$ harmonic and $\theta_{h}$ the phase shift for harmonic $h$. Only the first two harmonic components $\left(n_{h}=2\right)$ are accounted for in the assessment according to HiVoSS. In the present work, the coefficients for vertical vibrations $\alpha_{1}$ and $\alpha_{2}$ are taken equal to 0.4 and 0.1 , respectively.

The forces due to a group of pedestrians are represented in a simplified way by an equivalent uniformly distributed load on the bridge deck. Different traffic classes (TCs) are introduced representing different pedestrian densities. In table 1, an overview is given of the classes according to the HiVoSS guideline.

\begin{tabular}{c|ccccc}
\hline Traffic Class (TC) & TC 1 & TC 2 & TC 3 & TC 4 & TC 5 \\
\hline$d\left[\#\right.$ pers. $\left./ \mathrm{m}^{2}\right]$ & 15 pers. & 0.2 & 0.5 & 1.0 & 1.5 \\
\hline
\end{tabular}

Table 1: HiVoSS traffic classes and corresponding pedestrian densities.

The proposed load model consists of an equivalent number of perfectly synchronised pedestrians $N_{e q}$. A distinction is made between sparse and dense pedestrian densities. For low pedestrian densities, the pedestrians are assumed to move independently from each other and the equivalent number depends on the modal damping $\xi_{j}$. For higher pedestrian densities, the walking behaviour of the pedestrians is obstructed and the level of synchronisation increases. The higher level of synchronisation results in an increased number of equivalent pedestrians. $N_{e q}$ is found as follows:

$$
\begin{array}{cc}
N_{e q}=10.8 \sqrt{\xi_{j} N} & \text { for } d<1 \text { pers. } / \mathrm{m}^{2} \\
N_{e q}=1.85 \sqrt{N} & \text { for } d \geq 1 \text { pers. } / \mathrm{m}^{2}
\end{array}
$$

The amplitude of the equivalent uniformly distributed load $q_{e q}\left[\mathrm{~N} / \mathrm{m}^{2}\right]$ is calculated as:

$$
q_{e q}=\frac{N_{e q}}{S} \alpha_{h} G \psi\left(f_{j}\right)
$$

with $S$ the surface of the bridge deck and $\psi$ a reduction factor that accounts for the possibility of resonance as a function of the natural frequency $f_{j}$ of the bridge (figure 3(a)). Note that, due to the values of $\alpha_{1}$ and $\alpha_{2}$ assumed in this work, the reduction factor equals one at its maximum point for both first and second harmonic, whereas in the HiVoSS guideline, different maxima are found for both harmonics. If there is a considerable risk for resonance with the first or second harmonic of the load, a verification of the response is required. Due to the additional mass of pedestrians, the natural frequencies of the structure changes for different pedestrian densities. The reduction factor $\psi$ therefore depends on the traffic class. It should be noted that the reduction factor is very sensitive to small shifts in the natural frequency. 
The projection of the external force on mode $j$ can now be written as follows:

$$
F_{j}=q_{e q} \sum_{k=1}^{n_{\mathrm{eff}}} a_{\mathrm{eff}, k}\left|\phi_{j, k}\right|
$$

with $\phi_{j}$ the vector containing the mass-normalised modal displacements of the $j$-th mode for all nodes $n_{\text {eff }}$ of the effective bridge deck area $A_{\text {eff }}, a_{\text {eff }}$ the vector containing the bridge deck area allocated to the corresponding nodes (with $A_{\text {eff }}=\sum_{k=1}^{n_{\text {eff }}} a_{\text {eff }, k}$ ).

The maximum acceleration $\ddot{u}_{j, \max }$ assuming resonance with mode $j$ and disregarding the contribution from other modes is calculated as follows:

$$
\ddot{u}_{j, \max }=\frac{F_{j}}{2 \xi_{j}} \max \left|\phi_{j}\right|
$$

with $\xi_{j}$ the modal damping. Equation (6) shows that the final response is very sensitive to the modal damping $\xi_{j}$. A good estimation thus is essential for the response calculation. Suggested values for the modal damping are given by the guideline and can be used in design stage. After construction, measurements can be performed for verification.

The predicted value $\ddot{u}_{j, \max }$ is subsequently compared to thresholds corresponding to a comfort scale (maximal, mean, minimal comfort or unacceptable). Note that the vibration serviceability assessment must be performed considering all modes with $f_{j} \leq 5 \mathrm{~Hz}$ for different pedestrian densities.

\subsection{Vibration serviceability assessment for the Phénix footbridge}

Because of the low natural frequency of the first mode of the Phénix footbridge $\left(f_{1}=1.65 \leq\right.$ $5 \mathrm{~Hz}$ ), resonance with the first or second harmonic of the walking force must be considered and a vibration serviceability assessment according to HiVoSS is required. For higher natural frequencies, no risk for resonance with the walking force is assumed to exist. The proposed modal damping for the steel bridges in HiVoSS is $0.4 \%$. To account for the added mass effect of the pedestrians, the modal parameters are recalculated for each traffic class. In table 2 , the modal parameters of the first mode are summarised for the different traffic classes.

\begin{tabular}{c|ccccc}
\hline & TC 1 & TC 2 & TC 3 & TC 4 & TC 5 \\
\hline$f_{1}[\mathrm{~Hz}]$ & 1.65 & 1.63 & 1.60 & 1.55 & 1.50 \\
$\xi_{1}[\%]$ & 0.4 & 0.4 & 0.4 & 0.4 & 0.4 \\
$m_{1}[\mathrm{~kg}]$ & 131570 & 134560 & 139928 & 148821 & 157721 \\
\hline
\end{tabular}

Table 2: Modal parameters of the first mode of the Phénix footbridge accounting for the added mass of the pedestrians.

The vibration serviceability assessment for the first mode is given in figure 4. For low pedestrian densities, a maximal level of vibration comfort is predicted. For higher densities, the predicted level is unacceptable for vibration comfort. The highest acceleration is predicted for a pedestrian density of 1 pers. $/ \mathrm{m}^{2}$ corresponding to the TC $4\left(\ddot{u}_{\max }=4.22 \mathrm{~m} / \mathrm{s}^{2}\right)$. Although the equivalent force is higher for TC 5 than for TC 4 , the resulting response is slightly lower because of the smaller mass-normalised modal displacement for TC 5. 


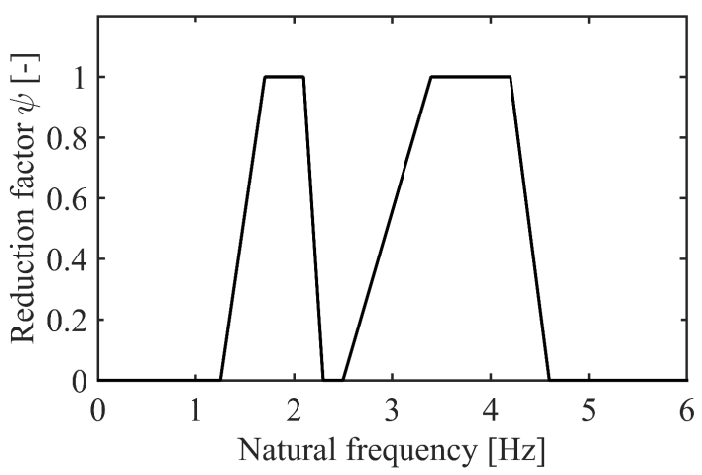

(a)

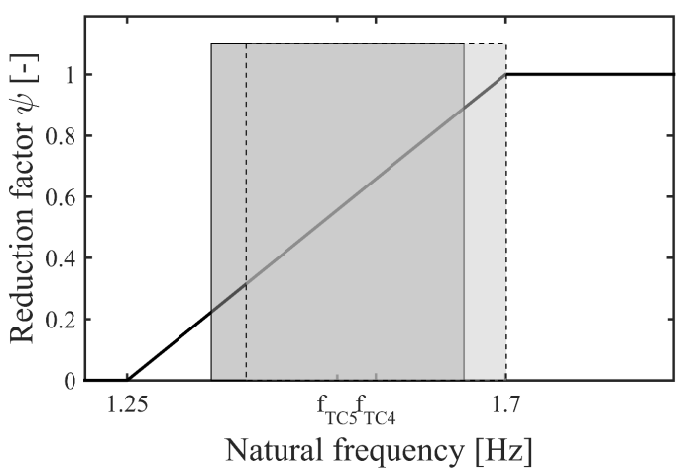

(b)

Figure 3: (a) Reduction factor $\psi\left(f_{j}\right)$ for vertical vibrations, (b) Zoom at reduction factor for TC 4 (light - -) and TC 5 (dark -) for the Phénix footbridge in a range of $10 \%$ about the first natural frequency of the bridge.

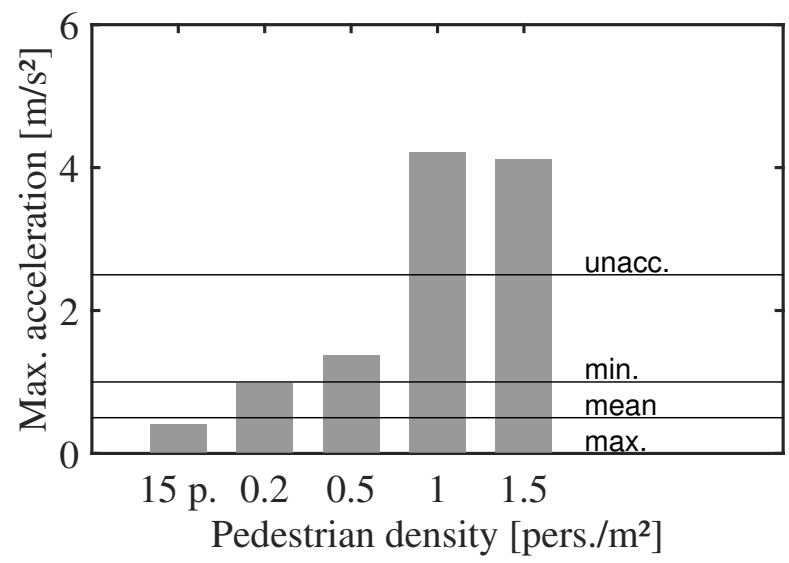

Figure 4: Vibration serviceability assessment of the first bending mode of the Phénix footbridge according to HiVoSS.

\section{EFFECT OF UNCERTAINTIES ON THE PREDICTED RESPONSE}

The vibration serviceability assessment as presented in the previous section is very sensitive to uncertainties on the natural frequency and modal damping. Various sources of uncertainty exist.

First, the effective stiffness of the footbridge may be uncertain due to a lack of knowledge on the material properties or the stiffness of the supports. Assumptions for Young's modulus in the finite element model may have a considerable influence on the effective stiffness. Time effects and degradation of the material properties also need to be considered. Young's modulus of concrete increases during the first months after construction [20]. From [21], it can be readily understood that the creep effects are larger for materials subjected to high temperatures for long time. The dynamic stiffness of bearing supports is given by the producer with corresponding tolerances. Bujňák et al. 222 reported that dripping water through open joints had damaged the bearing details significantly affecting the dynamic behaviour of the construction. The static and dynamic behaviour of the bearings may also differ as was illustrated in [23].

Intensive vibration monitoring of footbridges has also learnt that daily and seasonal temperature and environmental changes affect the natural frequencies [24, 25]. Natural frequencies generally 
decrease with increasing temperature [20]. Sohn in [26] states that temperature changes not only affect the stiffness but also the support conditions of the bridge. Similarly, Alampalli et al. [27] reported that frozen supports of the bridge resulted in an increased natural frequency. For a concrete slab bridge, Xia et al. [28] found a negative correlation between humidity and natural frequency but a positive correlation between humidity and modal damping.

In the present section, a fuzzy analysis is performed to investigate how uncertainties in the modal parameters affect the vibration serviceability assessment. The natural frequency and modal damping are modelled as convex fuzzy numbers. The uncertain variable is assumed to lay in an interval depending on the value of the membership function. For the natural frequencies, a maximal range of 0.9 to 1.1 times the nominal value is considered, in line with deviations recently observed in a large number of case studies [9]. For the modal damping, a larger interval of 0.5 to 1.5 times the proposed nominal value of $0.4 \%$ is adopted. For both variables, a triangular membership function is assumed, defined as:

$$
\begin{gathered}
\tilde{f}_{j}=\tilde{\Lambda}\left(f_{j, \text { nom }}, 0.9 f_{j, \text { nom }}, 1.1 f_{j, \text { nom }}\right) \\
\tilde{\xi}_{j}=\tilde{\Lambda}\left(\xi_{j, \text { nom }}, 0.5 \xi_{j, \text { nom }}, 1.5 \xi_{j, \text { nom }}\right)
\end{gathered}
$$

In figures 5(a) and 5(b), the membership function of the fuzzy variables is visualised. At different $\alpha$-levels, the corresponding limits of the interval can be found. In all analyses to come, the same fuzzy sets will be considered for the natural frequency and modal damping.

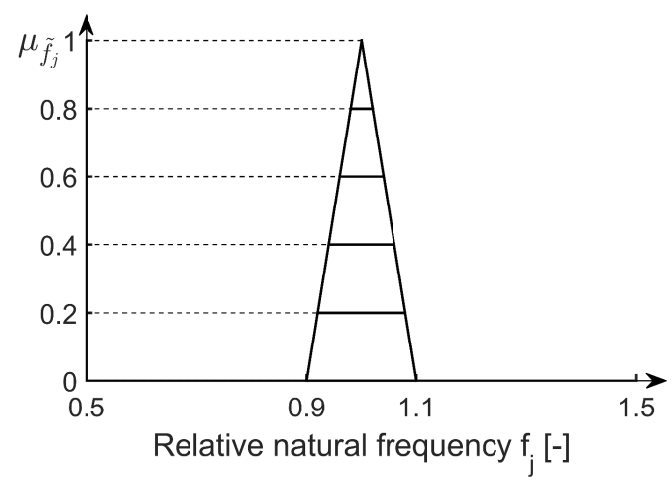

(a)

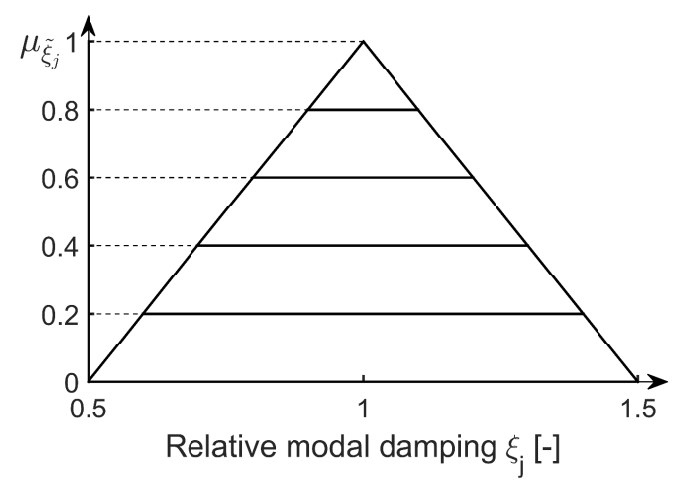

(b)

Figure 5: Membership function with discretisation in $\alpha$-cut sets for (a) relative natural frequency $f_{j}$ and (b) relative modal damping $\xi_{j}$.

The influence of these uncertainties on the vibration serviceability assessment of the Phénix footbridge is now investigated. It is assumed that there is no interaction between the uncertainties on the natural frequency and modal damping. The results of the vibration serviceability assessment for resonance with the first mode are given in figure 6 for different $\alpha$-levels. The assessment at $\alpha=1.0$ (darkest) corresponds with the assessment for the nominal values of the natural frequency and modal damping (figure 4). For low pedestrian densities, the predicted acceleration levels meet the minimal level for vibration comfort. For higher pedestrian densities, the acceleration levels are unacceptable for vibration comfort. For lower $\alpha$-levels, the maximum response level is higher due to the larger range of the natural frequency and modal damping. Since it cannot be guaranteed that the vibration serviceability criterion is satisfied for the assumed range of uncertainties on the natural frequency and modal damping, vibration reduction measures are needed. 


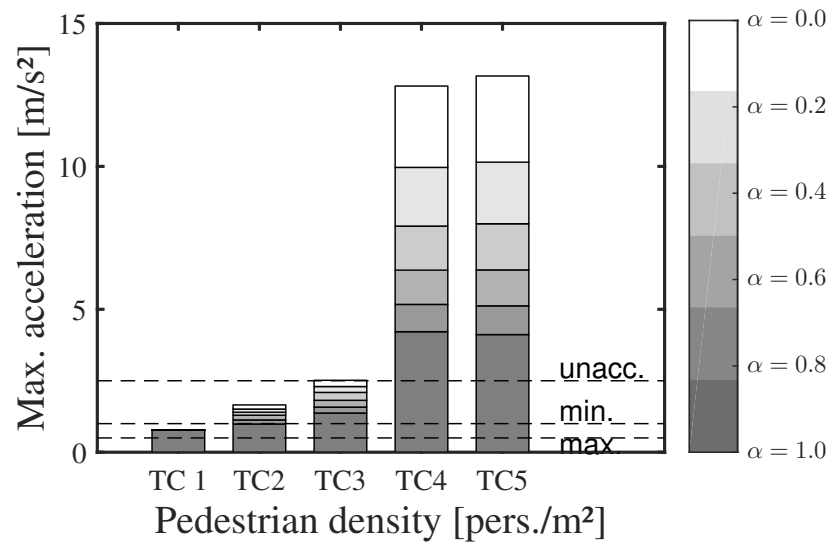

Figure 6: Vibration serviceability assessment for the Phénix footbridge considering resonance with the first bending mode: calculation of response for increasing levels of uncertainty for natural frequency and modal damping (dark to light).

\section{CONVENTIONAL TMD DESIGN}

\subsection{General formulation}

A TMD is frequently used to reduce the structural response in resonant conditions by increasing the damping. The TMD is defined by its mass $m_{\mathrm{TMD}}$, stiffness $k_{\mathrm{TMD}}$ and damping $c_{\mathrm{TMD}}$ characteristics. Since resonance is assumed, the footbridge equipped with TMD can be simplified as two coupled single degree of freedom (SDOF) systems. This implies that contributions from other modes are disregarded. The equivalent parameters for mode $j$ of the footbridge without TMD are written as follows:

$$
m_{j}=\frac{1}{\left(\max \left|\phi_{j}\right|\right)^{2}} \quad k_{j}=\left(2 \pi f_{j}\right)^{2} m_{j} \quad c_{j}=2 \xi_{j} m_{j}\left(2 \pi f_{j}\right)
$$

with $m_{j}$ the equivalent mass, $\max \left|\phi_{j}\right|$ the modal displacement corresponding to the TMD location, $k_{j}$ the equivalent stiffness and $c_{j}$ the equivalent damping for mode $j$. The dimensionless mass ratio $\mu$, the frequency ratio $\rho_{\text {TMD }}$ and damping ratio $\xi_{\text {TMD }}$ of the TMD are defined as follows:

$$
\mu=\frac{m_{\mathrm{TMD}}}{m_{j}} \quad \rho_{\mathrm{TMD}}=\frac{f_{\mathrm{TMD}}}{f_{j}} \quad \xi_{\mathrm{TMD}}=\frac{c_{\mathrm{TMD}}}{2 m_{\mathrm{TMD}} \omega_{\mathrm{TMD}}}
$$

The design of the TMD encompasses a trade-off between performance and cost. The design can be described as an optimisation problem where a cost function is to be minimised satisfying a set of constraints. In the present work, the cost function is the total cost of the TMD, which is assumed to depend linearly on the TMD mass $m_{\mathrm{TMD}}$. The constraint is a limit value $\ddot{u}_{\text {comfort }}$ for the maximal acceleration while the design variables are the TMD mass, stiffness and damping constant. The response is calculated for a 2DOF-system with the external force according to equation (5). A linear behaviour of the structure and TMD here is assumed [19]. An additional constraint is added to limit the relative displacement of the TMD $\Delta_{\text {rel }}$ between the TMD and the bridge deck $\left(\Delta_{\mathrm{rel}}=\left|u_{\mathrm{TMD}}-u_{\mathrm{DECK}}\right|\right)$ to a tolerable value $\Delta_{\mathrm{tol}}$ for constructability. The problem is formulated as follows:

$$
\begin{array}{lr}
\underset{m_{\mathrm{TMD}}, k_{\mathrm{TMD}}, c_{\mathrm{TMD}}}{\operatorname{minimise}} m_{\mathrm{TMD}} & \\
\text { subject to } & \ddot{u}_{\text {max }} \leq \ddot{u}_{\text {comfort }} \\
& \Delta_{\text {rel }} \leq \Delta_{\text {tol }}
\end{array}
$$


The widely used formulae provided by the classical work by Den Hartog [10] provide the optimal values of the stiffness and damping which minimise the displacement response for a given TMD mass and an undamped main structure. The expressions by Asami [11] generalise those given in the classical work by Den Hartog for a lowly damped structure. Alternative expressions in case of a damped main structure are presented by Ghosh and Basu in [29]. The optimisation problem (11) is now solved based on the analytical expressions by Asami [12, 30] which give the values of the TMD parameters $k_{\mathrm{TMD}}$ and $c_{\mathrm{TMD}}$ for a given TMD mass $m_{\mathrm{TMD}}$ by minimising the acceleration:

$$
\begin{array}{r}
\rho_{\mathrm{TMD}}^{*}=\sqrt{\frac{1}{1+\mu}} \\
\xi_{\mathrm{TMD}}^{*}=\sqrt{\frac{3 \mu}{8(1+\mu)}} \sqrt{1+\frac{27}{32} \mu}
\end{array}
$$

In this way, both constraints of the optimisation problem (11) can be verified for a range of masses $m_{\mathrm{TMD}}$, to identify the minimal value at which the constraints are satisfied.

\subsection{Conventional TMD design for the Phénix footbridge}

The vibration serviceability assessment presented in figure 4 shows that there is a high risk for resonance of the walking load with the first mode of the Phénix footbridge. The predicted acceleration levels for the considered mode clearly exceed the minimum level for vibration comfort. Figure 6 shows that the vibration levels are much higher for a reasonable range of uncertainties on the natural frequency and modal damping. In order to meet the vibration serviceability requirements, a TMD is now added at the midpoint of the bridge where the modal displacement of the first mode is largest.

The limit values $\ddot{u}_{\text {comfort }}$ and $\Delta_{\text {tol }}$ for the maximal vibration level and maximal relative displacement are chosen as $1.00 \mathrm{~m} / \mathrm{s}^{2}$ and $45 \mathrm{~mm}$, respectively.

Since the optimal frequency ratio $\rho_{\mathrm{TMD}}^{*}$ and damping ratio $\xi_{\mathrm{TMD}}^{*}$ depend on the mass ratio $\mu$ through equations $(12)$ and $(13)$, the two design constraints can be evaluated for a range of values of the mass ratio $\mu$. Figure 7 presents results for two cases. In the first case, the TMD parameters are tuned considering the nominal values of the modal parameters. In the second case, the TMD is tuned to the modal parameters leading to the highest response. A nominal design for the natural frequency and modal damping resulting in the highest response is considered.

In the vibration serviceability assessment based on the nominal modal parameters (figure 4), the largest acceleration value is found for TC 4 . To reduce this value to the limit value of $1 \mathrm{~m} / \mathrm{s}^{2}$, an effective reduction by a factor of 4.22 is required, corresponding to an effective damping $\xi_{\text {eff }}$ of $4.22 \cdot \xi_{1}=4.22 \cdot 0.4 \% \approx 1.7 \%$ related to maximum amplification factor of an SDOF system [31. Figure $7(\mathrm{a})$ shows that a value of $\mu=0.15 \%$ allows obtaining an effective damping of $1.7 \%$ for a nominal value of $0.4 \%$ for the modal damping. Verifying the second design criterion, a $\mu$-value of $0.74 \%$ is found to reduce the maximal relative displacement to $45 \mathrm{~mm}$ for the nominal design parameters (figure $7(\mathrm{~b})$ ) and an external force which can be deduced from equation (5) for the nominal value of the natural frequency for TC4 $\left(f_{1}=f_{1, \text { nom }}, \xi_{1}=\xi_{1, \text { nom }}\right)$.

Figure 6 shows that when deviations from the nominal values of the natural frequency and modal damping are considered, the acceleration level can reach $13.2 \mathrm{~m} / \mathrm{s}^{2}$ for TC 5 . Although a slightly lower level is found for the pedestrian density of TC 4, the latter is assumed for the TMD design to enable comparison with the previous case. The effect of the uncertain natural frequency for TC 4 is higher than for TC 5 as can be evaluated by the $\psi$-reduction factor at figure 3(b) where an uncertainty range of $10 \%$ of the nominal value of the natural frequency is considered. 
The TMD will be designed following Asami [12] but now with values of the natural frequency and modal damping of the main structure leading to the maximal acceleration levels found in figure 6. This is for $f_{1}=1.1 f_{1, \text { nom }}$ and for $\xi_{1}=0.5 \xi_{1, \mathrm{nom}}$. In this case, an effective damping of $12.8 \times\left(0.5 \xi_{1, \text { nom }}\right) \approx 2.56 \%$ is needed or a value $\mu=0.46 \%$. The second design constraint requires a mass ratio of $\mu=0.99 \%$.

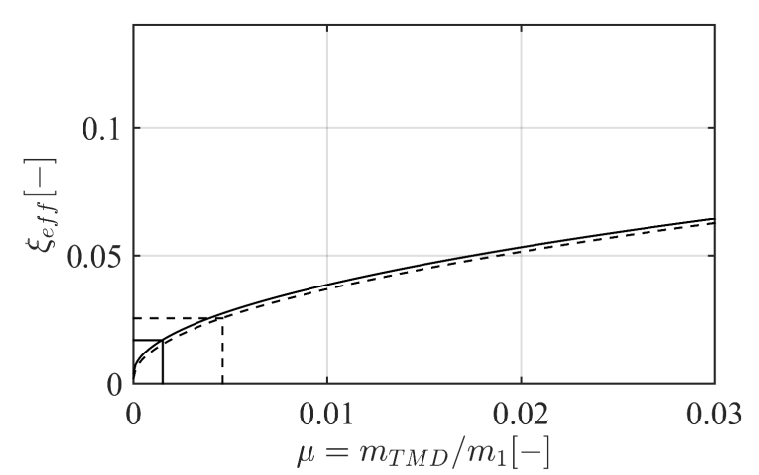

(a)

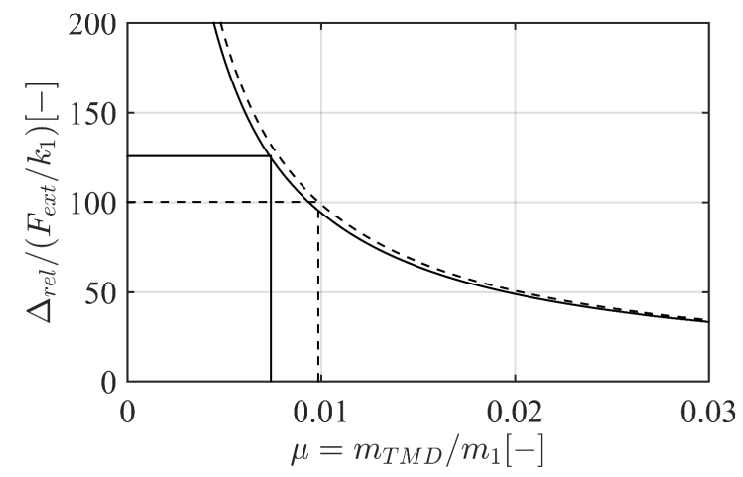

(b)

Figure 7: (a) Effective damping $\xi_{\text {eff }}$ and (b) ratio of the relative displacement and static displacement of the structure as a function of the mass ratio $\mu$ for a TMD designed according to Asami 12. Structure with modal damping $\xi_{1}=0.5 \xi_{1, \mathrm{nom}}=0.2 \%(--)$ and $\xi_{1}=\xi_{1, \mathrm{nom}}=0.4 \%(-)$. Minimally required mass ratio $\mu$ for nominal design $(-)$ and for design with natural frequency and modal damping leading to the highest acceleration $(--)$.

The optimal TMD parameters for both cases are presented in table 3 in absolute terms as well as in dimensionless values.

\begin{tabular}{c|ccc|ccc}
\hline & $m_{\mathrm{TMD}}[\mathrm{kg}]$ & $k_{\mathrm{TMD}}[\mathrm{N} / \mathrm{m}]$ & $c_{\mathrm{TMD}}[\mathrm{Ns} / \mathrm{m}]$ & $\mu[\%]$ & $\rho_{\mathrm{TMD}}[-]$ & $\xi_{\mathrm{TMD}}[\%]$ \\
\hline nominal & 1101 & 103191 & 1122 & 0.74 & 0.996 & 5.26 \\
worst-case & 1473 & 166630 & 1908 & 0.99 & 0.995 & 6.07 \\
\hline
\end{tabular}

Table 3: Optimal TMD parameters [12]: for nominal design and for design with natural frequency and modal damping leading to the highest accelerations.

\subsection{Effect of detuning}

The acceleration response for TC 4 is given in figures $8(\mathrm{a})$ and $8(\mathrm{~b})$ for the structure with TMD designed for the nominal case and the case resulting in the highest accelerations, respectively. The design constraint of $1 \mathrm{~m} / \mathrm{s}^{2}$ is not active as the constraint for the relative displacement is limiting in this case. When the acceleration response of the structure with TMD is evaluated for a range of uncertainties of the natural frequency and modal damping of the main structure as was introduced in section 4 (for $\alpha=0.0$ ), it becomes clear that the vibration serviceability criterion is not satisfied for the entire range of modal parameters considered. This is confirmed by the enveloping curve of the response for the considered uncertainty intervals. The response of the structure with TMD tuned at the bridge modal parameters resulting in the highest accelerations is given in figure 8(b). In this case, an acceleration level of $12.8 \mathrm{~m} / \mathrm{s}^{2}$ was reached without TMD. Again, it is observed how the design constraint is only satisfied for a small range of values about the nominal natural frequency and modal damping. The TMD designed for the highest value of the accelerations is in this particular case more robust with respect to uncertainties. This is due to the higher mass and damping of the TMD resulting in a higher response reduction. For the particular case, the highest 
acceleration for a system without TMD is predicted for the largest value of the natural frequency, i.e. $1.1 \times f_{1, \text { nom }}$ for TC 4 (see also $3(\mathrm{~b})$ ). If the TMD, tuned at the highest natural frequency, is evaluated for the whole range of frequencies, i.e. from $0.9 \times f_{1 \text {,nom }}$ to $1.1 \times f_{1 \text {,nom }}$, it is found that the enveloping response is less sensitive to the varying frequency in this particular case.

The constraint on the relative displacement of the TMD is evaluated in figures $9(\mathrm{a})$ and $9(\mathrm{~b})$ for both designs and the range of uncertainties. The maximal value approximately equals $45 \mathrm{~mm}$ for both designs as this is the limiting constraint. The small difference with $\Delta_{\text {tol }}$ is due to the assumptions and simplifications of the proposed method. The influence of the considered uncertainty intervals is larger for the TMD tuned at the nominal values of the natural frequency and modal damping.

The vibration serviceability assessment for the structure with TMD is now performed for all previously considered pedestrian densities (table 1). The assessment of the structure with the TMD tuned at nominal values is given in figure $10(\mathrm{a})$. For the nominal values of the modal parameters, the acceleration constraint is satisfied for all traffic classes while the influence of uncertainties results in lower levels of vibration comfort. In figure 10(b), the vibration serviceability assessment is given for the structure equipped with the TMD tuned at the natural frequency and modal damping resulting in the highest accelerations. It is here found that the acceleration constraint is not satisfied for the nominal values of the modal parameters because the TMD is detuned for that values. The sensitiveness to uncertainties in the natural frequency and modal damping of the footbridge is smaller in comparison with the design for the nominal modal parameters.

These results clearly show how the analytical expressions by Asami allow the designer to optimally tune a damper device for a given structure with known modal parameters. However, it does not provide any robustness with respect to uncertainties on the modal parameters of the structure, which are unavoidable in design stage.

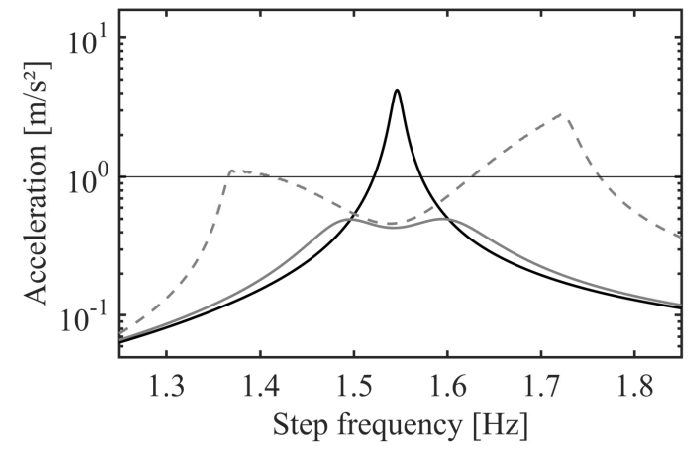

(a)

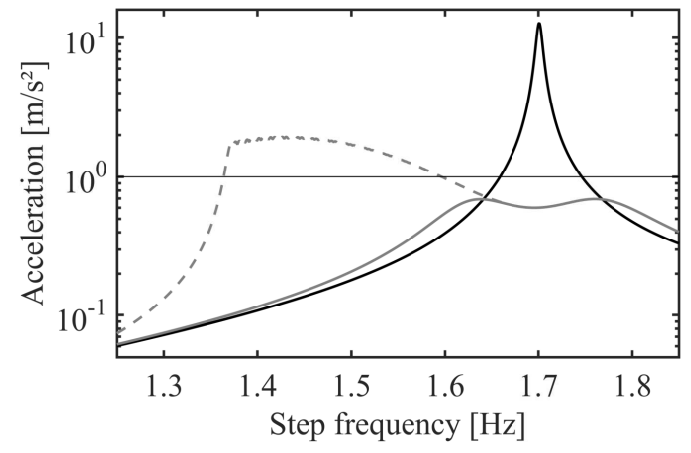

(b)

Figure 8: Maximal acceleration as a function of the step frequency for the structure without TMD (dark -) and with TMD tuned for (a) $f_{1, \text { nom }}$ and $\xi_{1, \text { nom }}$ and (b) $1.1 \times f_{1, \text { nom }}$ and $0.5 \times \xi_{1, \text { nom }}$ resulting in the highest acceleration (light -). Envelope of maximal response for all possible value of natural frequency and modal damping within the range corresponding to $\alpha=0.0$ (light - -) with loading according to TC 4 . Horizontal line: $\ddot{u}_{\text {comfort }}$ value. 


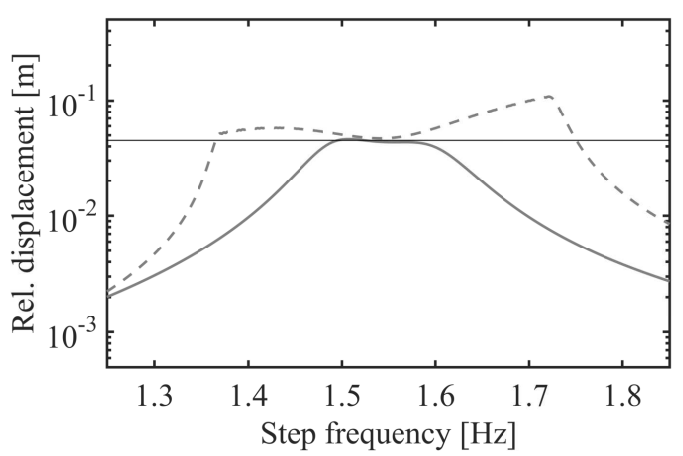

(a)

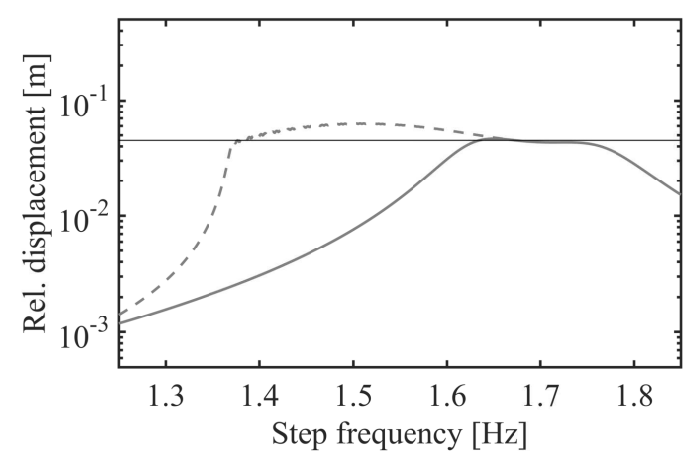

(b)

Figure 9: Maximal relative displacement as a function of the step frequency for the structure with TMD tuned for (a) $f_{1, \text { nom }}$ and $\xi_{1 \text {,nom }}$ and (b) $1.1 \times f_{1 \text {,nom }}$ and $0.5 \times \xi_{1 \text {,nom }}$ resulting in the highest acceleration (-). Envelope of maximal response for all possible value of natural frequency and modal damping within the range corresponding to $\alpha=0.0(--)$ with loading according to TC 4 . Horizontal line: $\Delta_{\text {tol }}$ value.

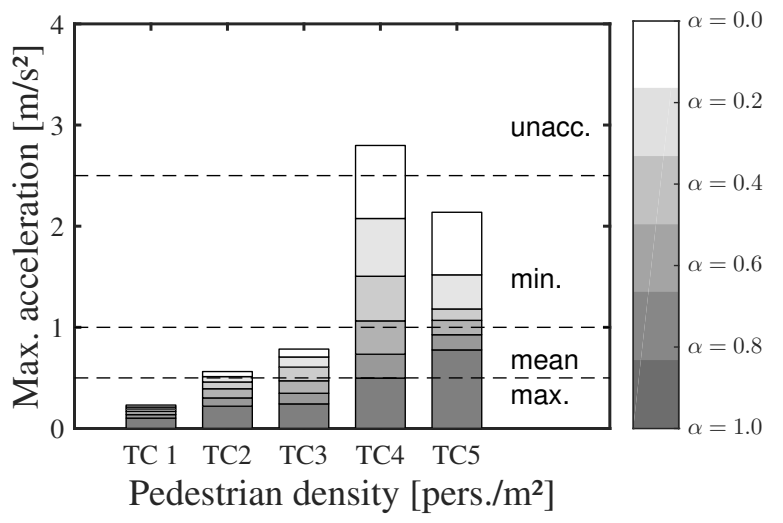

(a)

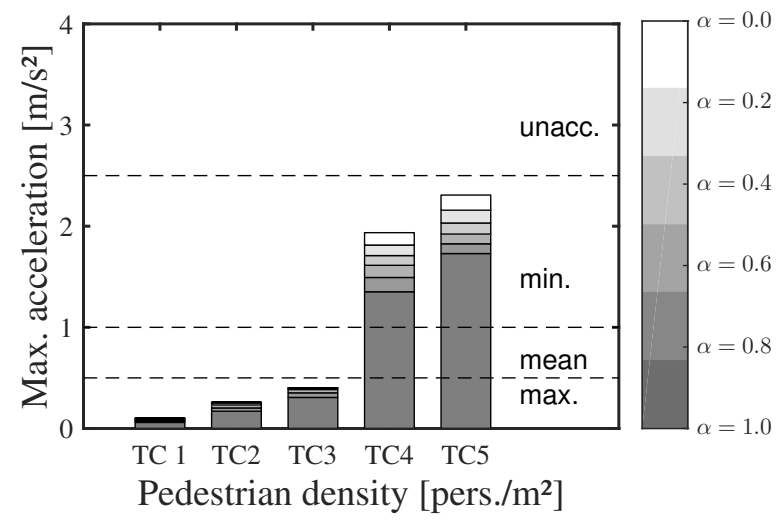

(b)

Figure 10: Vibration serviceability assessment for a structure with TMD tuned at (a) nominal parameters of natural frequency and modal damping, (b) natural frequency and modal damping resulting in highest accelerations. Calculation of response for increasing levels of uncertainty for natural frequency and modal damping (dark to light).

\section{TMD DESIGN BY ROBUST OPTIMISATION}

\subsection{Problem formulation}

This paper proposes a worst-case approach for robust optimisation of the TMD design. The trade-off between performance and cost is included in the optimisation problem where a cost function is to be minimised satisfying a set of constraints. In the present work, the cost function is the total cost of the TMD, which is assumed to depend linearly on the TMD mass $m_{\mathrm{TMD}}$. A multi-interval analysis is adopted to evaluate the trade-off between costs and robustness. For each interval, a deterministic optimisation problem is solved by minimising the TMD mass.

The limit value of $1 \mathrm{~m} / \mathrm{s}^{2}$ is also here chosen as the maximal allowed vertical acceleration $\ddot{u}_{\text {comfort }}$ and is implemented as a first constraint. For each traffic class, the same limit value is considered here. Alternatively, the acceleration constraint could depend on the pedestrian density. The maximal allowed relative displacement $\Delta_{\text {tol }}$ is set to a tolerable value of $45 \mathrm{~mm}$ and implemented 
as a second constraint. An upper and lower limit for the stiffness $k_{\mathrm{TMD}}$ and damping $c_{\mathrm{TMD}}$ of the TMD is included to ensure buildability. To take into account the influence of the uncertain natural frequency and modal damping, the response must satisfy the constraints for each pedestrian density and for all possible values of the natural frequency and modal damping in the considered interval. This leads to the following formulation of the optimisation problem at each $\alpha$-level in the analysis:

$$
\begin{aligned}
& \underset{m_{\mathrm{TMD}}, k_{\mathrm{TMD}}, c_{\mathrm{TMD}}}{\operatorname{minimise}} m_{\mathrm{TMD}} \\
& \text { subject to } \\
& \max _{\xi_{1}^{\alpha}, f_{1}^{\alpha}, \mathrm{TC}_{i}}\left(\ddot{u}_{\max }\right) \leq \ddot{u}_{\text {comfort }} \\
& \max _{\xi_{1}^{\alpha}, f_{1}^{\alpha}, \mathrm{TC}_{i}}\left(\Delta_{\mathrm{rel}}\right) \leq \Delta_{\text {tol }} \\
& k_{\min } \leq k_{\mathrm{TMD}} \leq k_{\max } \\
& c_{\min } \leq c_{\mathrm{TMD}} \leq c_{\max }
\end{aligned}
$$

with the limit values of the constraints summarised in table 4. The optimisation problem was solved using a sequential quadratic programming (SQP) algorithm as implemented in the Matlab function fmincon. The solution was verified by restarting the algorithm from different initial points. The range for $f_{1}^{\alpha}$ and $\xi_{1}^{\alpha}$ depends on the $\alpha$-level as shown in figures $5(\mathrm{a})$ and $5(\mathrm{~b})$.

\begin{tabular}{cl}
\hline$\ddot{u}_{\text {comfort }}$ & $1.00 \mathrm{~m} / \mathrm{s}^{2}$ \\
$\Delta_{\text {tol }}$ & $45 \mathrm{~mm}$ \\
$k_{\mathrm{TMD}}$ & {$\left[0-10^{6}\right] \mathrm{N} / \mathrm{m}$} \\
$c_{\mathrm{TMD}}$ & {$\left[0-10^{5}\right] \mathrm{Ns} / \mathrm{m}$} \\
\hline
\end{tabular}

Table 4: Constraints TMD optimisation problem for the Phénix footbridge

\subsection{Results}

The solution of the optimisation problem for the TMD design is given in table 5 for different levels of uncertainty. A linear behaviour of the TMD and main structure is assumed for this particular case [19. Table 5 summarises the parameters of the TMD, both in absolute terms and in dimensionless values, the range of the maximum peak accelerations and the range of the relative displacements for the traffic class $\mathrm{TC}_{i}$ that provides the active constraint which is determining for the solution. The TMD parameters were tuned, however, to satisfy the constraints for all traffic classes. The values for $\mu, \rho_{\text {TMD }}$ and $\xi_{\text {TMD }}$ are defined based on the nominal values of $f_{1}$ and $\xi_{1}$ as initial design point.

At the level $\alpha=1.0$, the nominal TMD design is found. In comparison with the nominal conventional design (table 3), a lower TMD mass is found (-10\%). The effect of the reduced TMD mass is compensated by a significantly higher TMD damping $(+109 \%)$ for $\alpha=1.0$. Both the acceleration and relative displacement constraint are active.

For a decreasing value of $\alpha$, the level of uncertainty increases and the minimally required TMD mass increases illustrating the trade-off between cost and robustness. The highest acceleration for a structure with TMD with minimised mass for TC 5 does not longer occur for the highest relative frequency as could be expected from figure 3(b). A strong increase of $m_{\mathrm{TMD}}$ is found from $\alpha=0.4 \rightarrow 0.2$ and from $\alpha=0.2 \rightarrow 0.0$ where the limit on the acceleration response for TC 4 becomes active for a structure with the highest natural frequency in the considered interval. The TMD damping $c_{\mathrm{TMD}}$ increases in absolute terms with the level of uncertainty but the corresponding dimensionless value $\xi_{\text {TмD }}$ slightly decreases. If needed, additional constraints can be implemented to 
limit the TMD mass and damping for activation of the TMD. The range of the peak accelerations and peak relative displacement also increases for a lower $\alpha$ because of the wider range for $\xi_{1}^{\alpha}$ and $\psi$ (due to $f_{1}^{\alpha}$ ). The larger the TMD mass, the smaller the relative displacement is.

At the level $\alpha=0.0$ corresponding to the largest range of uncertainties, both the TMD mass $(+34 \%)$ and damping $(+129 \%)$ are much higher in comparison with the conventional design (table 3). For the levels $\alpha=0.2$ and $\alpha=0.0$, the constraint on the displacement is not active because the mass needed to satisfy the constraint on the acceleration is already sufficiently high to satisfy both constraints. The maximal peak relative displacement is lower than $\Delta_{\text {tol }}$ for the highest levels of uncertainty.

\begin{tabular}{ccc|ccc|c|ccc|cc}
\hline$\alpha$ & $\begin{array}{c}f_{1}^{\alpha} \\
{\left[\times \mathrm{f}_{1, \mathrm{nom}}\right]}\end{array}$ & $\begin{array}{c}\xi_{1}^{\alpha} \\
{\left[\times \xi_{1, \mathrm{nom}}\right]}\end{array}$ & $\begin{array}{c}m_{\mathrm{TMD}} \\
{[\mathrm{kg}]}\end{array}$ & $\begin{array}{c}k_{\mathrm{TMD}} \\
{[\mathrm{N} / \mathrm{m}]}\end{array}$ & $\begin{array}{c}c_{\mathrm{TMD}} \\
{[\mathrm{Ns} / \mathrm{m}]}\end{array}$ & $\mathrm{TC}$ & $\begin{array}{c}\mu \\
{[\%]}\end{array}$ & $\begin{array}{c}\rho_{\text {TMD }} \\
{[-]}\end{array}$ & $\begin{array}{c}\xi_{\text {TMD }} \\
{[\%]}\end{array}$ & $\begin{array}{c}\ddot{u}_{\text {max }} \\
{\left[\mathrm{m} / \mathrm{s}^{2}\right]}\end{array}$ & $\begin{array}{c}\Delta_{\text {rel }} \\
{[\mathrm{mm}]}\end{array}$ \\
\hline 1.0 & {$[1.00-1.00]$} & {$[1.0-1.0]$} & 989 & 87821 & 2340 & 5 & 0.63 & 1.00 & 12.55 & $1.00-1.00$ & $45-45$ \\
0.8 & {$[0.98-1.02]$} & {$[0.9-1.1]$} & 1133 & 1004005 & 2628 & 5 & 0.72 & 1.02 & 12.11 & $0.86-1.00$ & $38-45$ \\
0.6 & {$[0.96-1.04]$} & {$[0.8-1.2]$} & 1265 & 120503 & 2880 & 5 & 0.80 & 1.04 & 11.66 & $0.84-1.00$ & $33-45$ \\
0.4 & {$[0.94-1.06]$} & {$[0.7-1.3]$} & 1396 & 136515 & 2964 & 5 & 0.88 & 1.05 & 10.74 & $0.81-1.00$ & $28-45$ \\
0.2 & {$[0.92-1.08]$} & {$[0.6-1.4]$} & 1656 & 164717 & 3334 & 4 & 1.11 & 1.03 & 10.10 & $0.59-1.00$ & $23-40$ \\
0.0 & {$[0.90-1.10]$} & {$[0.5-1.5]$} & 1976 & 201694 & 4366 & 4 & 1.33 & 1.04 & 10.94 & $0.57-1.00$ & $17-36$ \\
\hline
\end{tabular}

Table 5: Results multi-interval TMD optimisation: TMD optimal parameters and constraint evaluation at different $\alpha$-levels.

\subsection{Discussion}

The constraints are now evaluated at each $\alpha$-level to verify the performance of the robust TMD design. Next to evaluation of the response according to HiVoSS, an alternative evaluation of the TMD efficiency under pedestrian traffic for the Phénix footbridge is discussed in [19].

\section{Acceleration response}

The first design constraint concerns the maximal response level. At all $\alpha$-levels, the acceleration constraint is active for both TC 4 and TC 5. The acceleration response of a structure equipped with TMD is plotted for the nominal values of the natural frequency and modal damping in figures 11(a) (TC 4) and 11(b) (TC 5) and for the values of the natural frequency and modal damping resulting in the highest accelerations (worst-case scenario) in figures 11 (c) (TC 4) and 11(d) (TC 5). The enveloping curve of the response is plotted considering increasing levels of uncertainty. The nominal response clearly satisfies the constraint for the accelerations at all levels of uncertainty (figures 11(a) and 11(b)p. In the worst-case scenario, the response reaches the predefined comfort level (figures $11(\mathrm{c})$ and 11(d)] . 
TC 4

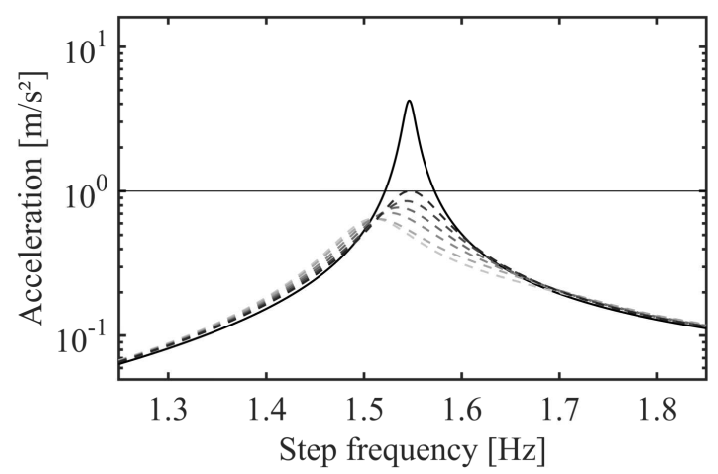

(a)

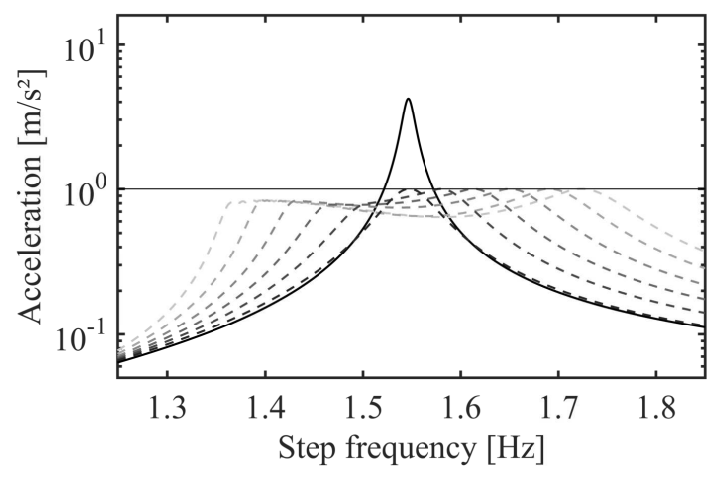

(c)

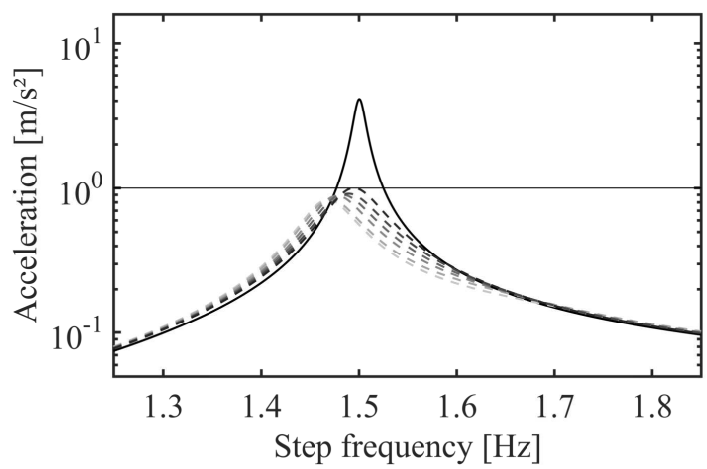

(b)

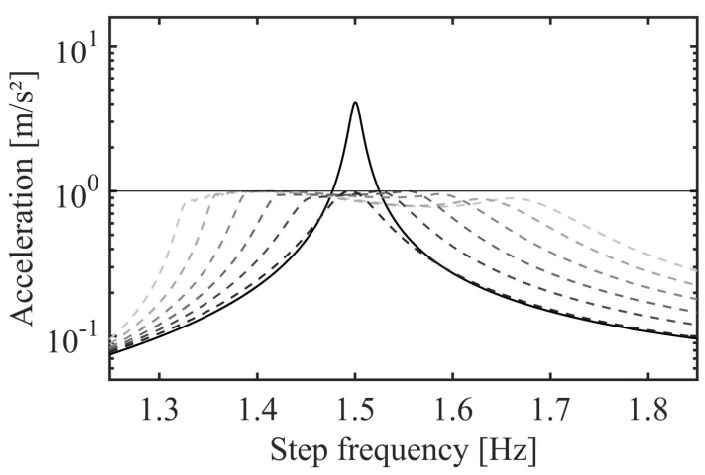

(d)

Figure 11: Maximal acceleration as a function of the step frequency for the structure without TMD (-), with optimised TMD (- $)$ for increasing levels of uncertainty $(\alpha=1.0-0.8-0.6-0.4-0.2-0.0$, dark to light): Acceleration response at nominal values of natural frequency and modal damping with loading according to: (a) TC 4, (b) TC 5. Envelope of maximal response for all possible values of natural frequency and modal damping within the range corresponding to each $\alpha$-level and loading according to: (c) TC 4, (d) TC 5. The horizontal line represents the maximal allowed acceleration limit of $1 \mathrm{~m} / \mathrm{s}^{2}$.

\section{Relative displacements}

The second design constraint is inactive for TC 4 and active in the range $\alpha=0.4 \rightarrow 1.0$ for TC 5. In figure 12(a), the relative displacement of the TMD is given for the nominal values of the natural frequency and modal damping for the structure with TMD tuned for each $\alpha$-level. The enveloping curves of response of the relative displacements considering the range of parameters at each level of uncertainty are given in figure $12(\mathrm{c})$, The limit value is never reached for TC 4 . The constraint is active for TC 5 as can be seen on figures 12(b) and 12(d). In the worst-case scenario, $\Delta_{\text {tol }}$ (horizontal line) is reached in the range $\alpha=0.4 \rightarrow 1.0$. 
TC 4

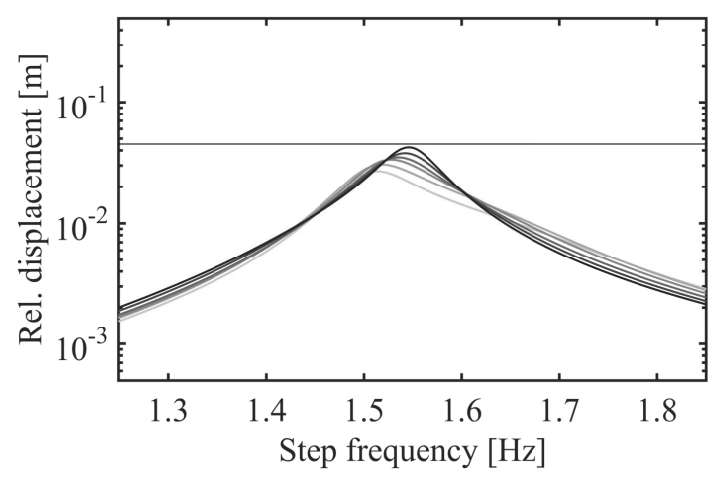

(a)

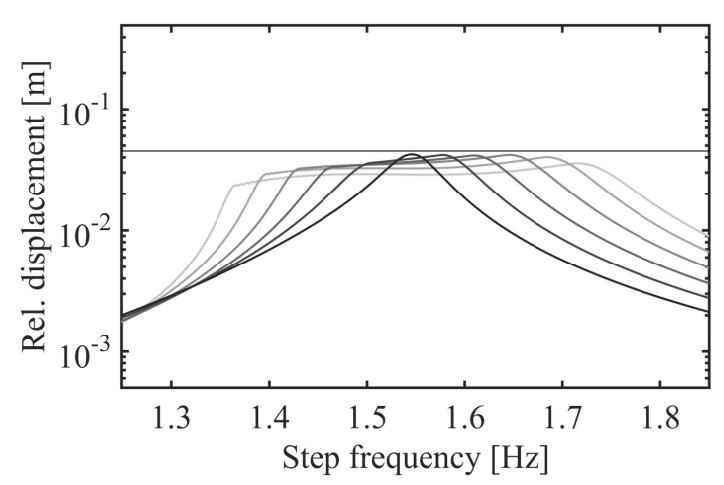

(c)
TC 5

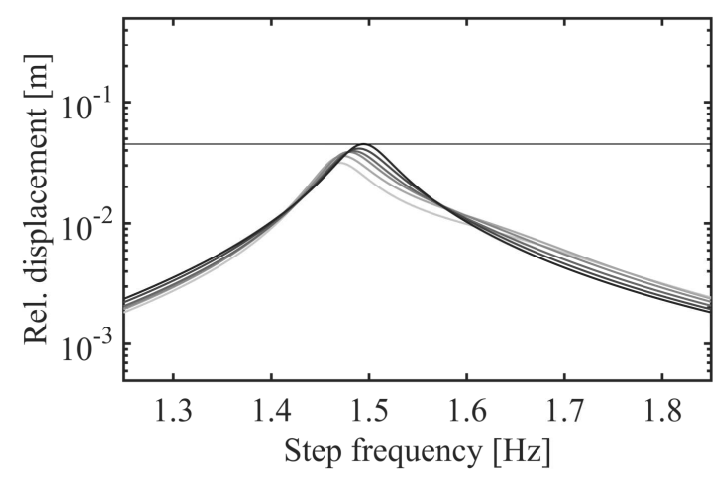

(b)

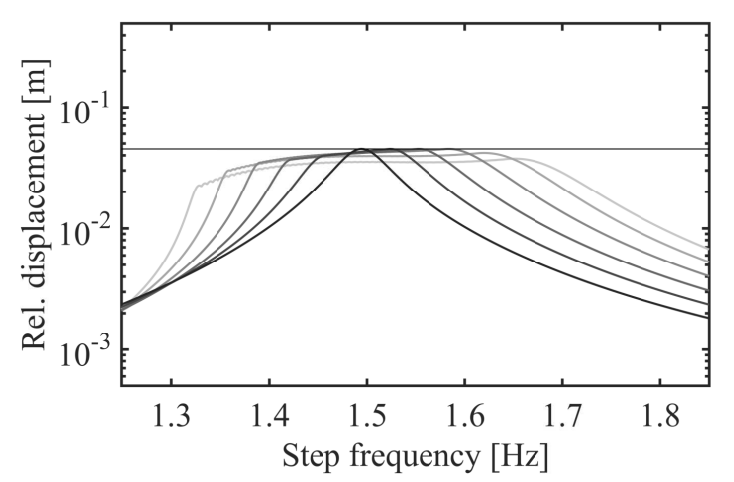

(d)

Figure 12: Maximal relative displacement as a function of the step frequency for the structure with TMD tuned at each $\alpha$-level $(\alpha=1.0-0.8-0.6-0.4-0.2-0.0$, from dark to light): Relative displacement response at nominal values of natural frequency and modal damping with loading according to: (a) TC 4, (b) TC 5. Envelope of maximal response for all possible values of natural frequency and modal damping within the range corresponding to each $\alpha$-level and loading according to: (c) TC 4, (d) TC 5. The horizontal line represents the maximal allowed relative displacement limit $\Delta_{\text {tol }}$ of $45 \mathrm{~mm}$.

\section{Vibration serviceability assessment}

The vibration serviceability assessment for a structure with TMD can be performed at each $\alpha$-level. The assessment at level $\alpha=0.0$ is given in figure $13(\mathrm{a})$ considering the largest range of uncertainties. The assessment for the nominal values of the natural frequency and modal damping is given by the darkest bar, while the influence of uncertainties is visualised by the lighter bars. The minimum level of vibration comfort now is guaranteed for each traffic class. A comparison between figures 10(b) and 13(a) clearly highlights the robust character of the optimised design.

In figure 13(b), the assessment is given for the structure with TMD tuned for level $\alpha=0.6$. The acceleration constraint is satisfied for the levels in the range $\alpha=0.6-1.0$ while for higher levels of uncertainty, the minimum level of vibration comfort is not guaranteed. 


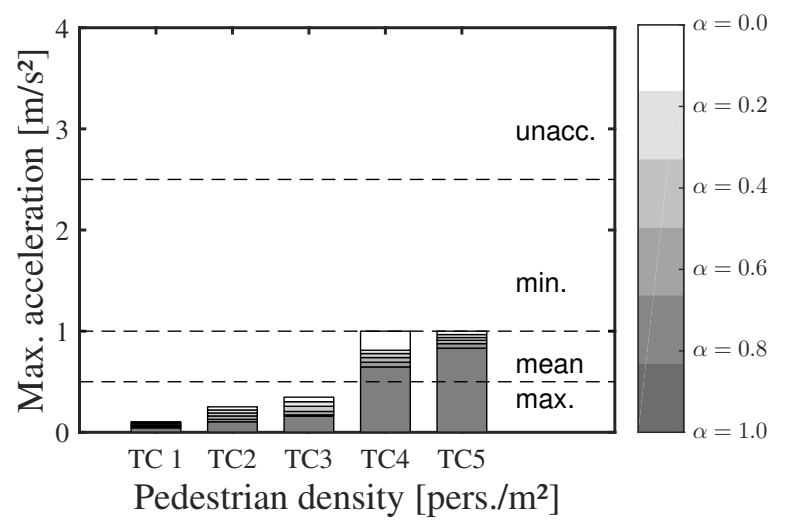

(a)

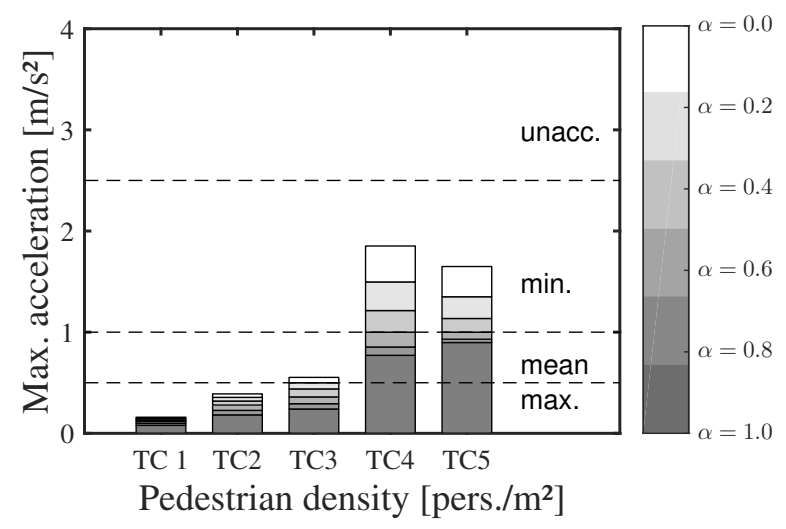

(b)

Figure 13: Vibration serviceability assessment for a structure with TMD tuned at natural frequency and modal damping resulting in highest response (a) for $\alpha=0.0$ considering the largest range of uncertainty, (b) for $\alpha=0.6$ considering a smaller range of uncertainty. Calculation of response for increasing levels of uncertainty for natural frequency and modal damping (dark to light).

\section{CONCLUSIONS}

The response of footbridges to human-induced excitation is highly sensitive to uncertainties on the modal parameters of the structure. The same holds for the performance of vibration reduction measures such as TMDs which are tuned at the modal parameters of the structure to maximise their performance.

A robust TMD design optimisation approach considering uncertainties on the modal parameters of the structure was therefore proposed. The natural frequency and modal damping were modelled as fuzzy numbers. Different levels of uncertainty were considered to investigate the trade-off between cost and robustness. By minimising a cost function, the TMD mass, stiffness and damping were tuned for the worst-case scenario at the different levels of uncertainties. It was found that the TMD mass and damping increase with the a higher level of uncertainty.

A comparison between the TMD tuned at the nominal values of the modal parameters and the conventional TMD has revealed that the TMD mass can be further reduced without violating the design constraints resulting in a more cost-effective design when it is assumed that the TMD mass determines the cost. For the TMD tuned at the largest level of uncertainties, a strongly increased mass and damping were found in comparison with the conventional design. By accounting for uncertainties in the vibration serviceability assessment for different traffic classes, it is guaranteed that the TMD performs satisfactorily in uncertain circumstances. The proposed method was illustrated using the HiVoSS guideline but it is generic and can be easily adapted for other vibration serviceability assessment methods.

\section{Acknowledgements}

This research is funded by the Agency for Innovation by Science and Technology in Flanders (IWT). Their financial support is gratefully acknowledged. 


\section{References}

[1] P. Van den Broeck, K. Van Nimmen, B. Gezels, E. Reynders, G. De Roeck, Measurement and simulation of the human-induced vibrations of a footbridge, in: Proceedings of the 8th International Conference on Structural Dynamics of EURODYN, Leuven, Belgium, 2011, pp. 941-948.

[2] S. Živanović, A. Pavic, P. Reynolds, Vibration serviceability of footbridges under human-induced excitation: a literature review, Journal of Sound and Vibration 279 (1-2) (2005) 1-74.

[3] E. Caetano, Á. Cunha, F. Magalhães, C. Moutinho, Studies for controlling human-induced vibration of the Pedro e Inês footbridge, Portugal. Part 2: Implementation of tuned mass dampers, Engineering Structures 32 (2010) 1082-1091.

[4] R. Rana, T. Soong, Parametric study and simplified design of tuned mass dampers, Engineering Structures 20 (3) (1998) 193-204.

[5] H. Werkle, C. Butz, R. Tatar, Effectiveness of "Detuned" TMD's for Beam-Like Footbridges, Advances in Structural Engineering 16 (1) (2013) 21-32.

[6] H. Jensen, M. Setareh, R. Peek, TMDs for Vibration Control of systems with Uncertain Properties, Journal of Structural Engineering 118 (12) (1992) 3285-3296.

[7] C. Petersen, Schwingungsdämpfer im Ingenieurbau, Maurer Söhne GmbH \& Company KG, 2001.

[8] E. Rizzi, D. Brescianini, M. Scotti, On the optimal tuning of tuned mass dampers in structural systems, Engineering Structures 20 (3) (1998) 193-204.

[9] K. Van Nimmen, G. Lombaert, G. De Roeck, P. Van den Broeck, Vibration serviceability of footbridges: Evaluation of the current codes of practice, Engineering Structures 59 (2014) 448-461.

[10] J. P. Den Hartog, Mechanical Vibrations, Dover, 1985.

[11] T. Asami, O. Nishihara, A. M. Baz, Analytical solutions to $\mathrm{H} \infty$ and $\mathrm{H} 2$ optimization of dynamic vibration absorbers attached to damped linear systems, Journal of Vibration and Acoustics 124 (2) (2002) $284-295$.

[12] T. Asami, O. Nishihara, Exact solution to $\mathrm{H} \infty$ optimization of dynamic vibration absorbers (application to different transfer functions and damping systems, Journal of Vibration and Acoustics 125 (3) (2003) 398-405.

[13] G. C. Marano, R. Greco, S. Sgobba, A comparison between different robust optimum design approaches: application to tuned mass dampers, Probabilistic Engineering Mechanics 25 (1) (2010) 108-118.

[14] C. Zang, M. Friswell, J. Mottershead, A review of robust optimal design and its application in dynamics, Computers \& Structures 83 (4) (2005) 315-326.

[15] G. C. Marano, G. Quaranta, Robust optimum criteria for tuned mass dampers in fuzzy environments, Applied Soft Computing 9 (4) (2009) 1232-1243.

[16] G. C. Marano, R. Greco, Robust optimization for TMD with uncertain bounded system parameters and stochastic excitation, Asian Journal of Civil Engineering (Building and Housing) 9 (5) (2008) 433-455.

[17] T. Igusa, A. Der Kiureghian, Response of uncertain systems to stochastic excitation, Journal of Engineering Mechanics 114 (5) (1988) 812-832.

[18] Research Fund for Coal and Steel, HiVoSS: Design of Footbridges (2008).

[19] K. Van Nimmen, P. Verbeke, G. Lombaert, G. De Roeck, P. Van den Broeck, Numerical and experimental evaluation of the dynamic performance of a footbridge with tuned mass dampers, Journal of Bridge Engineeringdoi:10.1061/(ASCE)BE.1943-5592.0000815.

[20] B. Peeters, G. De Roeck, One-year monitoring of the Z24-bridge: environmental effects versus damage events, Earthquake Engineering \& Structural Dynamics 30 (2) (2001) 149-171.

[21] P. J. Mendes, J. A. Barros, J. M. Sena-Cruz, M. Taheri, Development of a pedestrian bridge with GFRP profiles and fiber reinforced self-compacting concrete deck, Composite Structures 93 (11) (2011) 2969-2982.

[22] J. Bujňák, R. Hlinka, J. Odrobiňák, J. Vičan, Diagnostics and evaluation of footbridges, Procedia Engineering 40 (2012) 56-61.

[23] P. Verbeke, C. Schauvliege, K. Van Nimmen, G. De Roeck, P. Van den Broeck, Berenkooi - Report Dynamics of Structures, Tech. rep., Department of Civil Engineering, KU Leuven (2013).

[24] P. Moser, B. Moaveni, Environmental effects on the identified natural frequencies of the Dowling Hall Footbridge, Mechanical Systems and Signal Processing 25 (7) (2011) 2336 - 2357.

[25] W.-H. Hu, C. Moutinho, E. Caetano, F. Magalhães, Á. Cunha, Continuous dynamic monitoring of a lively footbridge for serviceability assessment and damage detection, Mechanical Systems and Signal Processing 33 (0) (2012) $38-55$.

[26] H. Sohn, Effects of environmental and operational variability on structural health monitoring, Philosophical Transactions of the Royal Society A: Mathematical, Physical and Engineering Sciences 365 (1851) (2007) 539560 .

[27] S. Alampalli, Influence of in-service environment on modal parameters, in: Proceedings-SPIE The International Society For Optical Engineering, Vol. 1, Citeseer, 1998, pp. 111-116. 
[28] Y. Xia, H. Hao, G. Zanardo, A. Deeks, Long term vibration monitoring of an RC-slab: Temperature and humidity effect, Engineering Structures 28 (3) (2006) $441-452$.

[29] A. Ghosh, B. Basu, A closed-form optimal tuning criterion for TMD in damped structures, Structural Control and Health Monitoring 14 (4) (2007) 681-692.

[30] F. Weber, G. Feltrin, O. Huth, Guidelines for structural control, Structural Engineering Research Laboratory, Swiss Federal Laboratories for Materials Testing and Research, Dubendorf, Switzerland.

[31] B. Weber, G. Feltrin, Assessment of long-term behavior of tuned mass dampers by system identification, Engineering Structures 32 (11) (2010) 3670-3682. 